\title{
The Adult Respiratory Distress Syndrome: Volumetric Overload Shocks in Patho-Etiology, Correcting Errors and Misconceptions on Fluid Therapy, Vascular and Capillary Physiology
}

\author{
Ahmed N Ghanem* \\ Retired Consultant Urologist, Egypt \\ *Corresponding author: Ahmed N Ghanem, Retired Consultant Urologist, No1 President Mubarak Street, Mansoura 35511, Egypt
}

Submission: October 15, 2018; Published: November 09, 2018

\begin{abstract}
Introduction and objective: To report critical literature analysis that shows volumetric overload shock (VOS) is the real patho-etiology of the adult respiratory distress syndrome (ARDS) demonstrating multiple errors and misconceptions on fluid therapy that predisposes to VOS and ARDS.

Material and methods: The literature on ARDS and physiological law of starling is critically analyzed revealing the multiple errors and misconceptions prevailing in fluid therapy. Recent reports on VOS in the patho-etiology of ARDS are summarized.
\end{abstract}

Result: The literature on ARDS and physiological law of starling is critically analyzed revealing multiple errors and misconceptions. Starling's law is wrong as both of its forces do not work as proposed. Errors have been corrected and the hydrodynamics of porous orifice G tube are advanced as replacement for Starling's law. The evidence confirmed VOS induced by sodium-based fluids is the real patho-etiology of ARDS.

Conclusion: The critical literature analysis on ARDS and physiological law of Starling rectified many errors and misconceptions. The hydrodynamics of the $\mathrm{G}$ tube in a surrounding chamber $\mathrm{C}$ that mimics capillary-interstitial compartment shows a magnetic fluid shaped phenomenon that gives a real replacement for Starling's law for the capillary-interstitial fluid transfer. The VOS proved to be the real patho-etiology of ARDS.

Keywords: Shock; ARDS; Volumetric overload shocks; Starling's law; capillary-interstitial fluid transfer

Abbreviations: ARDS: The Adult Respiratory Distress Syndrome; MVOD/F: Multiple Vital Organ Dysfunction/Failure Syndrome; VO: Volumetric Overload; VOS: Volumetric Overload Shock; VOS1: Volumetric Overload Shock Type 1; VOS2: Volumetric Overload Shock Type 2; CVP: Central Venous Pressure; BP: Arterial Blood Pressure; BW: Body Weight; CVS: Cardiovascular System; ISF: Interstitial Fluid; HST: Hypertonic Sodium Therapy; G Tube: Porous Orifice Tube; C: Chamber Around the G Tube; G-C Circulation: The Circulation Phenomenon between the G Tube and the Surrounding Chamber; PP: Proximal Pressure; DP: Distal Pressure; LP: Lumen Dynamic Pressures Inside a Tube; FP: Flow Pressure Component of LP; SP: Side Pressure Component of LP

\section{Introduction}

The adult respiratory distress syndrome (ARDS) was first reported in 1967 [1] that affects hundreds of thousands of cases worldwide every year and is associated with substantial morbidity, cost and mortality [2,3]. In the first report [1], volumetric overload (VO) of 12-14L was documented in every case but later reports rarely incriminated VO in its patho-etiology [2,3]. With VO unsuspected, the results of both randomized controlled trials (RCT) [4] and systemic reviews [2] were inconclusive. The most recent RCT investigating fluid therapy in MVOD/F aimed at the first 7 postoperative days thus missed the initiating event of bolus fluid therapy given during resuscitation or surgery that established the condition in the first place. Another RCT never mentioned VO or increase in body weight hence the most recent systemic review totally overlooked VO as possible insult inducing ARDS or MVOD/F syndrome [3].

The reason for overlooking VO as cause of ARDS is the accumulation of clinical misconceptions based on erroneous Starling's law on the capillary-interstitial fluid transfer [4]. This has subtly misled physicians into infusing big bolus VO for treating true or presumed hypovolaemia causing hypotension inducing ARDS or MOVD/F syndrome. The nihilistic approach used here is based on reminders of basic physics and physiology facts, plausible clinical observations [5], physics research revealing a novel hydrodynamic phenomenon [6], and clinical research [7,8] on VO complicating fluid therapy started 33 years ago [5] and has continued until today [6-12]. This report advances the hypothesis of Vo over time as the causative insult of ARDS or MVOD/F. 


\section{Historical Background}

Ever since fluid therapy has proved life-saving for millions of poly-trauma victims of World War Two (WW2), the procedure was transferred into clinical practice with all its success and complications without verification. Reports from WW2 and clinical practice during the $3^{\text {rd }}$ quarter of the $20^{\text {th }}$ century demonstrate complications of fluid therapy that was reported as ARDS and is recognized today as the MVOD/F syndrome. The slogan of that era, that remains operative today, was: "Too much of a good thing must be a good thing"!? This is untrue particularly and obviously concerning water: water is essential for life, yet its excess or deficiency is equally detrimental or lethal. Excess kills by flooding/ drowning while lack is lethal by dehydration/ drought.

Fluid therapy is used in hospitals mainly for treating hypotension of haemorrhagic, hypovolaemic and septic shocks as well as in the resuscitation of poly-trauma and burns, preloading and peri-operative maintenance of prolonged major surgery $[3,13]$. This is when, where and how ARDS/MOVD/F is induced as iatrogenic complication of bolus VO used for treating hypotension of presumed or true volume deficit. It occurs only in hospitals, commonly on intensive care units (ICU) [13], never in community and thus is iatrogenic.

\section{The Scientific Basis of VO}

The blood volume of an adult is $5 \mathrm{l}$ and all body fluid volume and tonicity are precisely regulated and the cardiovascular system (CVS) capacity is 7l [14-16]. Trying to fit 10-15l of fluid in 7l capacity container, must spell fluid over! This basic physics principle should limit physiology laws on CVS volume-pressure relationship that govern fluid therapy. Thus, any infused bolus VO that exceeds CVS capacity must leak out into the interstitial fluid (ISF) space within minutes. Excess fluid pools in the third potential space of pleura, peritoneum and gut and some enter the intracellular space while the kidneys are trying to execrate the surplus, if it does not fail [79].

One current widely received misconception is that any infused VO of plasma albumen or substitute must stay intravascular according to Starling's law on capillary-interstitial fluid equilibrium. This issue has, long and repeatedly, been proved wrong in both physiology and clinical studies demonstrating that albumen does not work [17]. It has repeatedly called for re-consideration of Starling's law [18], yet it remains operative in clinical practice on fluid therapy!

\section{Supportive Clinical Evidence}

When there is true CVS volume deficit most of the infused fluid stays intravascular topping CVS volume up to normal level, while an excess VO distributes within minutes between the CVS and ISF spaces with excess fluid spelling over into the third space. While flooding of the ISF space manifest mainly as trunk edema, the excess intracellular fluid causes cell edema that does not manifest clinically. The cells become hypoxic manifesting with the clinical features of MVOD/F while flooded lung alveoli manifest with ARDS. Also, some edematous cells many disintegrate by hydrolysis leaking its contents into the serum identified later as the systemic inflammatory response syndrome (SIRS). Advances in ventilation have improved oxygen uptake at the lung alveoli, and advances of CVS and renal support have improved prognosis, prolonged survival and modified the clinical picture of ARDS. However, failure of transfer at the capillary-ISF and cellular levels remains as evidenced by the current prevalence of high morbidity and mortality of ARDS [1-3].

A large bolus of VO is a constant insult in all cases of ARDS while the quoted causes such as shock, burns and drowning are predisposing conditions that indeed vary in severity and prognosis. In addition to the serious predisposing conditions, occurrence after prolonged major surgery and polytrauma resuscitation is also common $[2,3,17]$. Fluid preloading on induction of anaesthetia and replacing intra-operative loss are highly overestimated and the liberal fluid infusions or volume expansion for combating such hypotension are not evidence-based procedures. If this is appreciated and implemented by all physicians involved in fluid therapy and resuscitation, substantial reduction of ARDS and MVOD/F cases will occur, many lives will be saved, and the morbidity and cost will be substantially reduced.

The extra-vascular leakage of VO fluids into the ISF space is an internal flooding that cause the pathological torso and limb edema commonly seen on ICU [13] affecting all cases of ARDS and MOVD/F. The excess fluid is confirmed by increased body weight. Patients who die go to the mortuary with it and those who recover must lose it before discharge from ICU and hospital. Internal flooding and edema of vital organs are obvious on the postmortem examination [7-11].

The major concern, and worry, is that most involved physicians do not consider such gross torso edema pathological! Excess ISF is even thought advantageous on the erroneous belief that overhydration provides better oxygenation of tissues and cells! Such view overlooks the obvious difference between irrigation and flooding of a cell, organism or land that makes the difference between life and death. A patient on ICU with an excess of $7-14 \mathrm{~kg}$ of body fluids causing ISF, cell and vital organ edema cannot be considered as normal. The most harmful effect of VO flooding ISF space, however, is not the detectable subcutaneous edema but is the hidden edema affecting the vital organs manifesting clinically with MVOD/F syndrome. This is revealed clearly on postmortem examination [8] and may also be revealed on CAT and MRI.

The clinical severity of ARDS and MVOD/F depends not only on VO quantity but also on the time $(\mathrm{t})$ of gain. Severity is directly proportional to VO but inversely proportional to $t$. The fluid type and tonicity are also important. 


\section{What is VO?}

For simplicity and practicality reasons, VO complications of therapeutic fluids in clinical practice may be segregated into 2 groups based on the fluid type: sodium-free fluid (Type 1) or V01 that is characterized with dilutional hyponatremia and sodiumbased fluid (Type 2) or VO2 which has no clear serum marker. Both pathological VO groups may induce ARDS or MVOD/F on gaining varied quantities and demonstrate different hemodilution serum markers [7-9]. There is also minor variation among members of each group. Large infusion or absorption of the irrigating fluids during the transurethral resection of the prostate (TURP) surgery using 1.5\%Glycine, Mannitol or Sorbitol, and 5\% Dextrose infusion or parenteral nutrition are examples of V01 fluid $[6,8,9]$. A pathological V01 induces the TURP syndrome with its characteristic acute dilutional hyponatremia that ends up with the clinical manifestations of MVOD/F of which cerebral signs predominate $[5,7,8]$. Examples of VO2 fluids include normal saline, Hartmann's, Ringer's, plasma proteins, plasma albumin and substitutes and blood transfusions. These VO2 fluids have subtle serum markers. The pathological quantity of VO2 inducing MVOD/F is 2-3 times larger than V01.

On embarking on bolus volume expansion for the resuscitation of hypotension, it may be useful to consider the maximum capacitance of the vascular system of 71 that cannot be exceeded, and the maximum blood loss of trauma that is incompatible with life on arrival to a hospital is about its half that equal $\approx 3.51$. These figures of blood and plasma volumes represent $10 \%$ and $5 \%$ body weight (BW), respectively. The figure of 3.51 (5\%BW) with narrow deviation of (0.51) should limit the maximum volume to infuse after bleeding control. The same 3.51 (5\%BW) may be pathological VO inducing MVOD/F when give in one hour.

It is also worth noting that hemorrhagic shock has true blood volume deficit. A true fluid deficit also occurs in hypovolemic shock of burns, heat stroke and severe dehydration that require special careful assessment of the true deficit and meticulous replacement. In other causes of hypotension there is no real volume deficit but only mal-distribution of fluid between the vascular and ISF spaces or micro-vascular dilatation while the normal vascular volume remains there. Thus, hypotension is not synonymous with hypovolemia. Volumetric overload is known to exist in cardiogenic shock/failure where excess vascular volume and ISF edema and in which volume expansion is agreeably absolutely contra-indicated.

The precise figures of therapeutic fluid replacement and physiological fluid challenge versus the pathological VO need precise quantification and definition. What is ambiguously referred to as conservative versus liberal approaches of fluid therapy $[1,2]$, have wide personal and local variation. It is also of vital importance to recognize and identify the responses of therapeutic and physiological VO on one hand, and the paradoxical responses of pathological VO on the other, particularly its effect on the vascular pressures and renal function [8-10]. Acute renal failure
(ARF) is a feature of the TURP syndrome that is characterized by hyponatremia yet present with essentially most features of the MVOD/F syndrome $[3,8]$. These pathological responses on vascular pressure and renal function are exactly opposite of physiological responses of VO.

\section{Errors and Misconception on Fluid Therapy}

The errors and misconception that mislead physicians, anesthetists, Intensive care unit (ICU) specialists and resuscitation team using overzealous liberal volume expansion in treating recognized shocks are deeply rooted. Erroneous conceptions on vascular volume-pressure relationship on the arterial and venous sides of circulation are identified here.

\section{Error I}

Every arterial hypotension is considered synonymous with hypovolemia or at least treated as such with volume expansion in every clinical case of shock, anesthetic induction or operative period!

\section{Correction I}

Hypotension is not synonymous with hypovolemia. As mentioned above the cause of the primary recognized shock and hypotension must be differentiated. The difference between the therapeutic/ physiological VO regarding (quantity versus response) in contrast with the paradoxes of pathological VO on arterial pressure and renal response must be precisely identified. Two paradoxical responses of pathological VO require recognition: one is inducing hypotension shock and the second is causing ARF. The transition from the hypovolemic hypotension shock into the VO hypotension shock during overzealous volume expansion occurs seamlessly unnoticed and undetected by any monitoring until it manifests later on ICU with torso edema and increased BW of ARDS or MVOD/F patients.

\section{Error II}

The volume-pressure relationship of the vascular system is perceived as infinite strait line!?

\section{Correction II}

The volume-pressure relationship particularly that of vascular volume and arterial pressure is a limited line segment, beyond which the relation collapses. Within limits, increasing vascular volume (physiological or therapeutic VO) increases arterial pressure but when such limit is exceeded (pathological VO) a paradoxical hypotension occurs. A similar VO paradox exists on the renal function; while physiological VO induces diuresis a pathological VO causes ARF as part of the features of MVOD/F. These two paradoxes are not new but hardly recognized.

\section{Error III}

The central venous pressure (CVP) and pulmonary capillary wedge pressure (PCWP) as monitoring parameters guiding fluid 
therapy are given a value of 18 to $22 \mathrm{~cm}$ water as currently practiced on many ICUs $[3,13]$. Although current recommendations $[2,3]$ indicate that CVP and PCWP are unreliable and no longer being used, evidence from prevalence of ARDS and MOVD/F on ICU testify differently, and it remain part of its definition [2]. The confounded error underlying the misconception of high positive CVP is related to a deeply rooted physiological error.

\section{Correction III}

The given figures of CVP and PCWP are erroneously too high yet remain widely practiced. Persistence to achieve such high CVP using massive volume expansion is among the misleading reasons for inducing pathological VO causing ARDS. The infused fluid rapidly shifts out of the vascular system and CVP may drop back to below $10 \mathrm{~cm}$ water, then another bolus $\mathrm{VO}$ is given before the gross torso edema and increase of BW becomes obvious. The correct CVP figures are given in all physiology textbooks that swing around 0 (at mid-axillary line) with a range of +7 to $-7 \mathrm{~cm}$ water [14-16]. If we do not understand how Nature works, we must faithfully imitate until reliable methods of monitoring fluid therapy are found.

\section{Error IV}

The capillary forces responsible for irrigating and oxygenating the ISF space and cells are mixed up with that causing edema, flooding and drowning.

\section{Correction IV}

It is strongly recommended that every physician involved in fluid therapy, ARDS and MVOD/F management should reconsider what is the physiological function of the arterial and venous pressures and which pressure is responsible for what? In particular, relating the pathological ISF accumulation or subcutaneous edema with the forces on which the hypothesis that dictates capillary-ISF transfer on the causation of dropsy, proposed by Starling [4] at the Lancet in 1886, reveals the error. The reason is that the forces on which this hypothesis is based govern the volume and pressure regulation of the vascular and ISF compartments, and subsequently cell viability. Being false, this hypothesis underlies most mentioned erroneous concepts on fluid therapy. Starling's hypothesis was wrongly made later into physiological law [19]. It may be realized that this is the major error responsible for the current dilemma on ARDS and MOVD/F syndrome concealing its real patho-aetiology of $\mathrm{VO}$ [10-12].

\section{Error V}

The major misconception, and unfortunately the most prevailing, is wrongly assuming that the vascular system is an all positive pressure system, in which not only the mentioned arterial volume-pressure relationship is misconceived as infinite strait line but also keeping high venous pressure and ISF tissue over-hydrated are erroneously believed to enhance cell nourishment and oxygen delivery. This underlies the liberal volume expansion pumping in too much fluid that creates edema, flooding and drowning of the
ISF tissue as well as vital organs and cells! This is precisely the error underlying the pathological VO inducing ARDS and MVOD/F syndromes in current clinical practice.

\section{Correction V}

To assume that CVS to be an all positive pressure system is quite simply wrong. In fact, there is a lot of negative physiological pressure under the skin of many areas and organs of the body that should be kept that way- as this is how it functions best. It is well known that the pleural spaces have negative pressure and the pressure in alveoli alternates. The CVP of normal subjects may swing around Zero, between positive +7 and negative $-7 \mathrm{~cm}$ water [14-16]. The intracranial pressure is also negative. Thus, the ISF space of subcutaneous tissues, most organs and parts of the body have negative pressure of $-7 \mathrm{~cm}$ water that has been demonstrated [20] and re-affirmed [21] but neither considered nor satisfactorily explained.

There is nothing that can explain the negative pressure of the ISF space with efficient rapid irrigation, not edema, flooding and downing, except the negative energy phenomenon of the porous orifice tube [10-12]. The only high positive pressure of the circulatory system is the arterial pressure and this seems to be so for very good reason: it is the driving force for ejecting fluid through the capillary orifice creating the side negative energy pressure that drives the dynamic autonomous magnetic field-like fluid circulation between capillary lumen and surrounding tissues - keeping the ISF tissue pressure negative, appearing almost dry, while efficiently irrigated and oxygenated!

\section{Further Remarks on VO}

A therapeutic volume replacement of measured blood or fluid loss causing hypotension episode or shock must be precisely calculated and replaced avoiding over-estimation. A physiological VO added to the actual measured blood loss is perhaps the safest fluid regimen during major surgery. Physiological VO should be adequate to cover the insensible fluid loss from fasting to end of surgery. In other words, the safest maximum acute volume expansion should not exceed the capacitance of the vascular system by more than $1 \%$ BW. In situations where the loss is difficult to be accurately assessed, such as in polytrauma victims with internal cavity continuous bleeding and multiple fractures, it may be reasonably assessed in terms of maximum blood volume loss that is incompatible with life as basis for calculating the maximum volume replacement. In addition to clinical assessment, consider a normal recent BW and calculate the real total blood and plasma volume of such patient at base line and after resuscitation to measure VO.

To make it simple but accurate enough think of the vascular volume of $\approx 51$ and capacitance of CVS system of $\approx 7 \mathrm{l}$ of an adult. Its half equals the plasma volume of $\approx 3.5 \mathrm{l}$ that also approximately equals the daily fluid intake. Physiological bolus VO is about $1 / 3$ of the plasma volume $\approx 1-1.1667 \mathrm{l} /$ hour. This physiological VO plus the accurate therapeutic volume should be the maximum needed for 
resuscitation that should increase both the arterial blood pressure and urine output.

If the patient does not respond, consider either a concealed blood loss continuing that need control while fluid replacement is being done, or other cause of the hypotension shock such as microvascular dilatation of normo-volaemic patient, cardiac failure or a pathological VOS has already occurred. Except for cases with internal blood loss, an acute increase in BW is perhaps the best available for detecting pathological VO. If acute volume expansion increased BW by more than 5\% carry the risk of such pathological VO inducing ARDS or MVOD/F is real. The type and tonicity of fluid used as well as its quantity and time of VO gain is also considered [7-9].

Multiples of the bolus physiological VO in a normo-volaemic subject may become pathological with increasing degree of severity. A pathological VO of 3.51 in one hour induces moderate ARDS or MVOD/F and certainly $7 l$ is serious. These figures are accurate for V02 fluids. For pure V01 they are less by $\approx 1 / 3$. A bolus means rapid infusion of VO within $<1$ hour. When the figures are transferred into percentage of $\mathrm{BW}$, the plasma volume equals $\approx 3.51$ ( $5 \% \mathrm{BW}$ ) of $70 \mathrm{~kg}$ adult. A physiological VO equals $\approx 1 / 3$ of plasma volume $\approx$ $1-1.167$ l ( $\approx 1.67 \%$ BW). A pathological VO of $\approx 3 \%, 5 \%$ and $10 \%$ BW causes mild, moderate and severe ARDS or MVOD/F, respectively. The percentage figures apply to children and women also.

Should you wish to make it more challenging if the kidney remains functional, consider its maximum excretory ability in 1 hour, and subtract it from the gained VO, in order to determine the retained pathological VO. The next objective is to try to help the patient get rid of the retained VO surplus fluid within 24-48 hours while providing adequate ventilation and oxygenation, cardiac and vascular support, using diuretics and/or dialysis in cases of ARF. Hypertonic sodium therapy of $5 \% \mathrm{NaCl}$ or $8.4 \%$ sodium bicarbonate has proved lifesaving [5,7-12]. Extremes of age and women have poor tolerance to $\mathrm{VO}$ as they do to dehydration.

The type and tonicity of fluid affects clinical severity too. A pathological V01 acutely loading the vascular system with $>5 \% \mathrm{BW}$ causes serious morbidity, or even sudden death, and is characterized by the acute dilution hyponatremia $[22,23]$. It induces paradoxical hypotension shock and ARF [6-8]. This means that an acute change of the circulatory volume in either direction induces hypotension shock. The same VO of distilled water, still being used as irritant for the TURP surgery at some parts of the World, is probably lethal via sequelae of intravascular hemolysis. The same quantity of VO2 fluids $\approx 5 \%$ BW may cause subtle pathological changes but VO2 of $\approx 7-14 \mathrm{l}(10-20 \% \mathrm{BW})$ is that observed in severe cases of ARDS and MVOD/F [1].

Understanding the phenomenon of the porous orifice tube may help to rectify the errors and misconception on intravenous fluid therapy, redefine recognized shocks and identify the new VOS that resolve the riddle of the MVOD/F syndrome. No clinical RCT study will ever produce useful conclusions before the mentioned issues, errors and misconceptions are considered, stratified and rectified.

\section{Corrections of Errors and Misconceptions on Fluid}

\section{Therapy: Relevance To ARDS}

Two recently reported articles at The Lancet triggered the following comments. The first article is a comment updating perioperative fluid management [3] that faithfully reflected currently received views. However, it failed to identify an optimum regimen or define what is VO? The second article is a review [2] on acute lung injury and ARDS recognizing it as MVOD/F syndrome. It stated: "The importance of this syndrome with such staggering prevalence of morbidity and mortality was realized 25 years ago". The pointedout errors and misconceptions are self-evident while corrections are based on experience, clinical observations, research work and plausible overlooked documented evidence [7-11].

When the current rules on fluid therapy fail to provide adequate reliable guidance to practicing physicians perhaps a reliance on simple proven facts of physiological data, easily verifiable clinical observations and rejecting erroneous hypotheses may make better solid ground of evidence-based medicine for resolving such complex clinical dilemmas. The best executed prospective randomized controlled trials (RCT) and systemic reviews [2,3] will fail to give satisfactory answers or solutions.

\section{Volumetric Overload Shocks (VOS)}

Volumetric Overload Shock (VOS) is a condition caused by massive fluid infusions in a short time [7-9] and is of two types; Type one (VOS1) and Type two (VOS2). VOS1 is induced by sodium-free fluid gain of 3.5-5l in one hour such as Glycine, Glucose, Mannitol and Sorbitol. It is known as the TURP syndrome or hyponatremic shock [22] that was previously induced in dogs using 5\% Dextrose [23]. VOS2 is induced by massive infusion of sodium-based fluids such as normal saline, Ringer, Hartmann, plasma, plasma substitutes and blood transfusions that may complicate the therapy of VOS1. VOS2 also complicates fluid therapy in critically ill patients suffering from other known shocks such as hypovolemic, hemorrhagic and septic shocks and presents with ARDS. VOS2 is induced by the gain of 12-14l of sodium-based fluids when reported in ARDS [2]. The occurrence of massive interstitial tissue edema with congestion of vital organs, pleural and peritoneal effusions, in the presence of hypotension shock, casted doubt on Starling's law! These issues were investigated at the clinical and physiological/physical fronts [5-12].

Two clinical studies aiming to understand the TURP syndrome and recognizing VOS were done. A prospective clinical study on 100 consecutive TURP patients of whom the condition of TURP syndrome affected 10 patients with severe hypotension and bradycardia and severe acute dilution $\mathrm{HN}$ of $<120 \mathrm{mmol} / \mathrm{l}[5,7]$. Volumetric overload was the only significant factor in causing the condition. The second clinical study involved a case series of 23 cases of the TURP syndrome manifesting as VOS1 [8]. Volumetric overload quantity and type is shown in Figure 1 [8]. The first 3 cases 
died as they were diagnosed and treated erroneously as one of the recognized shocks and treated with further volume expansion. The remaining 20 patients were correctly diagnosed as VOS1 and treated with hypertonic sodium therapy (HST) of 5\% Sodium
Chloride or $8.4 \%$ Sodium Bicarbonate. Each patient passed $4-51$ of urine followed by recovery from shock and coma. This treatment was successful in curing all patients bringing them back from dead $[8,11]$.

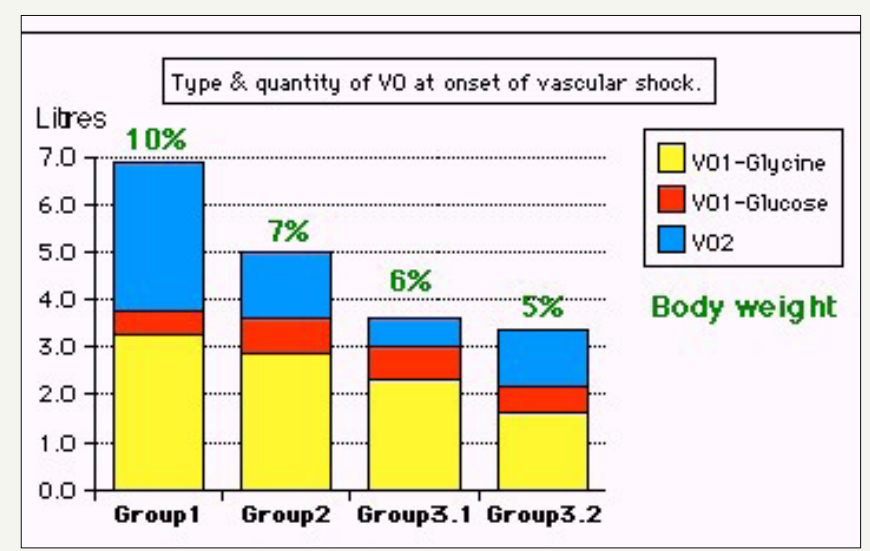

Figure 1: Shows volumetric overload (VO) quantity (in liters and as percent of body weight) and types of fluids. Group 1 was the 3 patients who died in the case series as they were misdiagnosed as one of the previously known shocks and treated with further volume expansion. Group 2 were 10 patients from the series who were correctly diagnosed as volumetric overload shock and treated with hypertonic sodium therapy (HST). Group 3 were 10 patients who were seen in the prospective study and subdivided into 2 groups; Group 3.1 of 5 patients treated with HST and Group 3.2 of 5 patients who were treated with guarded volume expansion using isotonic saline. (Reproduced with permission from reference [8]).

The physicalinvestigation involved studies of the hydrodynamics of the porous orifice $(G)$ tube comparing it to that of Poiseuille's tube $[6,10,11]$. Thousands of experimental measurements of pressures at various parts of a circulatory system incorporating the $G$ tube in a chamber to mimic the capillary-interstitial fluid compartment. The effect of changing the proximal (arterial), the distal (venous) pressures and the diameter of the inlet on side pressure of the $G$ tube and chamber pressure as well as the dynamic magnetic field like fluid circulation around the $\mathrm{G}$ tube were documented. It is quite remarkable how this circulatory model mimics the circulatory system in heath and disease. This dynamic magnetic field like fluid circulation around the $\mathrm{G}$ tube and surrounding it in $\mathrm{C}$ chamber provides adequate replacement for Starling's law. The physiological equivalent of this physical study was done on the hind limbs of sheep [10]. It demonstrated that arterial pressure causes suction not filtration due to the effect of pre-capillary sphincter. It is the only possible explanation why the interstitial tissue pressure is negative of $-7 \mathrm{~cm}$ water $[20,21]$. Venous pressure augmented filtration and edema or dropsy formation.

Shock is a disturbance at the capillary cellular level impairing the capillary-interstitial fluid transfer; hindering delivery of oxygen and removal of waste products. The process is also governed by Starling's law [5]. In this law the arterial pressure is considered the force causing capillary filtration! If this is true, how come that arterial hypertension though very common never causes edema? Starling based his hypothesis on Poiseuille work on strait uniform brass tubes [14]. Latter evidence however demonstrated that the capillary is a porous narrow orifice (G) tube as it has a precapillary sphincter [24] and pores that allow the passage of plasma proteins [25]. As the capillary pores allow the passage of plasma molecules, nullifying the osmotic pressure of plasma proteins i.e. oncotic pressure does not exist, a call for reconsideration of Starling's hypothesis was previously made [18] but there was no alternative at that time. This replacement came to light when the hydrodynamics of the $\mathrm{G}$ tube were discovered.

The hydrodynamics of the $G$ tube [7,11-13] (Figure 2) demonstrated that the proximal (arterial) pressure induces a negative side pressure gradient on the wall of the G tube causing suction most prominent over the proximal half and turns into positive pressure over the distal half. Incorporating the $G$ tube in a chamber $(\mathrm{C})$, representing the interstitial space surrounding a capillary, demonstrated a rapid dynamic magnetic field-like fluid circulation between the $\mathrm{C}$ and $\mathrm{G}$ tube lumen. This is a mixing engine between $\mathrm{C}$ and $\mathrm{G}$ effecting rapid irrigation under negative pressure i.e. without flooding or edema or dropsy formation. Incorporating the $\mathrm{G}$ tube and $\mathrm{C}$ in a circulatory model driven by electric pump inducing proximal pressure similar to arterial pressure; causing suction from $\mathrm{C}$ into the lumen of $\mathrm{G}$ tube. The distal (venous) pressure augments filtration. This proves that the arterial pressure causes suction not filtration at the capillary interstitial fluid circulation, and hence Starling's law is wrong. The reported hydrodynamics of the $\mathrm{G}$ tube provides an adequate mechanism for replacing Starling's law as the capillary interstitial fluid circulation. 


\section{Starling's Law}

It was mentioned that most errors and misconceptions on fluid therapy that mislead physicians into inducing VO causing ARDS and MVOD/F syndrome were deeply rooted to a faulty hypothesis on the capillary-interstitial fluid (ISF) transfer. Starling's hypothesis [4] is based on two main forces acting in opposite directions to move fluids between the capillary lumen and the ISF space namely; the hydrostatic and oncotic pressures. Both forces were based on physics experiments and the hypothesis was brought strait from physics to medicine at The Lancet in 1886. The hydrostatic pressure is the capillary arterial pressure presumed responsible for the force casing filtration pushing fluid out of the capillary lumen into the ISF space that is higher at the arterial end of the capillarycausing dropsy. This pressure force was based on Poiseuille's work on the dynamics of strait brass tube of uniform diameter [14]. It was, however, discovered 8 decades later, in 1967, that every capillary tube has a pre-capillary sphincter regulating its narrow orifice [24] of about $5 \mu \mathrm{m}$ compared to lumen diameter of $7 \mu \mathrm{m}$. This orifice proved to create totally different hydrodynamics [6] when compared to Poiseuille's tube.

\section{Why the Oncotic Pressure Does Not Work?}

The oncotic pressure is the presumed absorption force responsible for returning fluid back into the capillary lumen [4]. Again, this was based on physics experiment in which albumen was separated from water by a membrane impermeable to albumen molecules. That experiment [19], in 1948, showed an oncotic force of albumen but it was shown later that the oncotic force is too weak and too slow to be clinically solely responsible for fluid return from the interstitial fluid (ISF) space into the capillary tube lumen [26]. Also, in 1967, it was discovered that the capillary wall is a porous membrane [25] allowing easy free passage of plasma protein molecules in and out between the capillary lumen and ISF space, through these pores identified as the inter-cellular slits [25] The pressure in the ISF space was measured inside subcutaneously implanted perforated capsule and proved negative of $-7 \mathrm{~cm}$ water [20]. The negative ISF pressure was confirmed by similar study that also demonstrated that plasma protein molecules move freely between the implanted capsule and blood stream [21]. Thus, the normal capillary under physiological conditions is completely leaky to albumen after all! So, what does this mean?

Quite simply this means that clinically none of Starling forces work, certainly not in vivo! The oncotic force does not exist across porous leaky capillary membrane with large pores through which plasma proteins pass freely in either direction! Albumen has long been known not to work and that has repeatedly called for reconsideration of Starling hypothesis [18]. In the pulmonary circulation the arterial pressure is less than the oncotic pressure, and in the liver, lung and muscles, the plasma protein concentration may equal that of the plasma [15]. More recently the BMJ made a slogan out on it "Why albumen may not work?". This was based on Cochrane Injuries Group systemic review of RCT on ARDS and MVOD/F demonstrating that albumen does not work [17]. If one of the forces does not work the whole hypothesis must be faulty. Yet there was no alternative explanation or challenging hypothesis on how the capillary-ISF circulation really works that can replace that of Starling. Currently, however, there is one now [10-12].

\section{The Role of Arterial Pressure}

In Starling's law, the arterial pressure is presumed the main force responsible for capillary filtration and dropsy formation as stated in all physiology textbooks [14-16]. Evidence from daily clinical practice, however, confirms that this never happens! Although arterial hypertension is very common there is not a single case report in which arterial hypertension causes edema! The only proven well known cause of clinical edema is elevated venous pressure. So obviously in a dynamic circulatory system the venous and arterial pressures play totally different roles in the dynamics of capillary-ISF movements across the capillary wall! Adding to the fact that the physiological pressure of the ISF compartment is $-7 \mathrm{~cm}$ water $[20,21]$ that has never been considered or adequately explained. This negative ISF pressure reverts into positive value in pathological edema!

So, what is the exact role the arterial and venous pressures play in capillary-ISF transfer? This question was answered by physics experiments on porous orifice $(G)$ tube made on a macro scale to the capillary tube, tested initially alone and subsequently on enclosing it in a surrounding chamber (C) mimicking the ISF space [6,10-12]. The inflow or proximal pressure (PP) mimics arterial pressure and the outflow or distal pressure (DP) mimics the venous pressure. Based on a macro scale of the capillary, the G tube has $7 \mathrm{~mm}$ diameter with a varying orifice diameter of $2-6 \mathrm{~mm}$ at the inlet and holes in its wall. Results are summarized here.

The hydrodynamics of $\mathrm{G}$ tube was investigated between 1981-3, concluded in 1985, and repeated until 1987. The new hydrodynamic phenomenon was observed in 1983 (Figure 2). The inflow pressure on pushing fluid through the narrow orifice created fluid jet inside the $G$ tube lumen. This fluid jet has two dynamic component pressures, the flow pressure that is always positive and maximum near the inlet, and the side pressure that is a gradient maximally negative near the inlet and turns positive near the exit. The fluid jet on exerting negative side pressure gradient on the wall caused fluid suction into $G$ tube lumen over the proximal half that was maximal near the inlet. The side pressure gradient turned positive over the distal half of the G tube maximum near the exit. This pushes fluid out of the G tube lumen causing filtration mostly near the exit. On enclosing the $\mathrm{G}$ tube in a surrounding $\mathrm{C}$ chamber, mimicking ISF space, a hydrodynamic G-C circulation phenomenon between fluid in the $\mathrm{G}$ tube lumen and the surrounding chamber $\mathrm{C}$ occurred. 


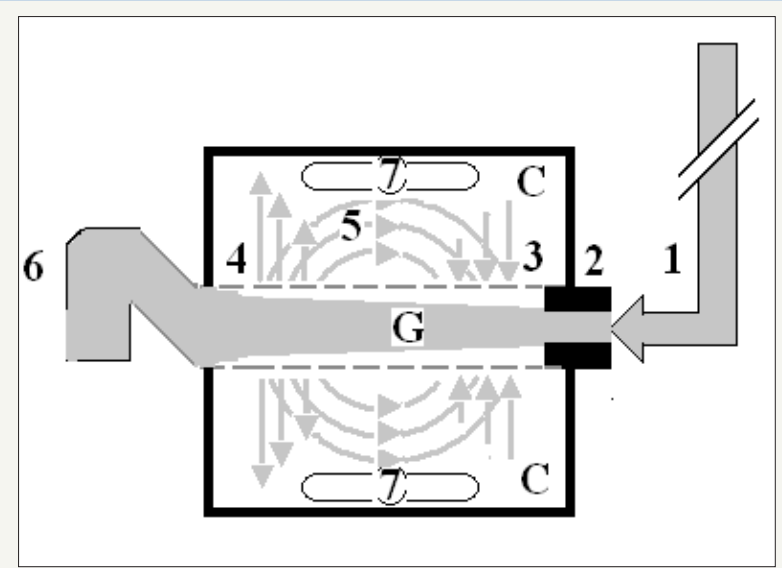

Figure 2: Shows Diagram of the porous orifice $(G)$ tube enclosed in chamber $(C)$ based on several photographs demonstrating the magnetic field-like G-C circulation phenomenon. The proximal inflow (arterial) pressure (1) pushes fluid through the orifice (2) creating fluid jet in the lumen of the G tube. The fluid jet creates negative side pressure gradient causing suction maximal over the proximal half of the G tube near the inlet (3) that sucks fluid into lumen. The side pressure gradient turns positive pushing fluid out of lumen over the distal half maximally near the outlet (4). Thus, the fluid around $\mathrm{G}$ tube inside $\mathrm{C}$ moves in magnetic field-like fluid circulation (5) taking an opposite direction to lumen flow of G. tube. The inflow (arterial) pressure (1) and orifice (2) induce the negative side pressure energy creating the dynamic G-C circulation phenomenon that is rapid, autonomous and efficient in moving fluid out from the G tube lumen at (4), irrigating $C$ at (5), then sucking it back again at (3), maintaining net negative energy pressure (7) inside C. The distal outflow (venous) pressure (6) enhances outflow at (4) and its elevation may turn the negative energy pressure (7) inside $\mathrm{C}$ into positive, increasing volume and pressure inside $\mathrm{C}$ chamber. (Reproduced with permission from reference [11]).

\section{The Phenomenon of The Porous Orifice (G) Tube}

The hydrodynamic phenomenon of the $\mathrm{G}$ tube is a rapid dynamic autonomous magnetic field-like fluid circulation rapidly circulating fluid between the $G$ tube lumen and surrounding $C$ chamber-the (G-C) circulation. Also, a net negative energy pressure is created in chamber $\mathrm{C}$, akin to ISF space. All the forces involved in creating the G-C circulation phenomenon were evaluated. The effect of the inflow proximal pressure (PP) and out flow distal pressure (DP) variations, and on varying the orifice diameter, on the negative side energy pressure gradient, chamber $\mathrm{C}$ pressure changes and the efficiency of the G-C circulation were evaluated in thousands of experiments $[6,11,12]$.

The hydrodynamics of the G tube were also contrasted to that of Poiseuille's tube of uniform lumen by simply removing the narrow orifice at the inlet or reversing it. The pressure exerted on the wall of Poiseuille's tube is always positive and is higher near the inlet pushing fluid out of the lumen with nothing to return it back- as happens in a high-pressure system such as when a garden hose or water supply pipe is punctured! On enclosing Poiseuille's tube in a chamber, the tube-chamber circulation reversed its direction taking the same direction of lumen flow and the pressure in the chamber became high positive and more fluid volume accumulated in chamber that became leaky.

In a porous orifice tube, however, thanks to the effect of the narrow orifice, the same inflow pressure exerts dynamic negative energy pressure gradient on the wall that sucks fluid into the tube lumen. As the side pressure gradient is turned positive near the exit, it pushes fluid out of the lumen. This creates the phenomenon of magnetic field-like fluid circulation around the tube with a net negative pressure in the surrounding chamber. The fluid in the chamber moves in an opposite direction to the main flow in porous orifice tube's lumen. Increasing the inflow pressure enhances the negative energy, negative side pressure, negative chamber pressure that speeds up the tube-chamber circulation, and vice versa. On increasing the outflow pressure, akin to venous pressure, it cancels the negative energy side pressure causing more fluid to move out of the porous tube's wall into the chamber- reverting chamber pressure into positive value.

The orifice plays vital unique role in the G-C circulation dynamics, speed and efficiency. A too narrow or too wide orifice slows down the tube-chamber circulation as does a drop in the inflow pressure and elevation of distal outflow pressure. Such changes can simulate the capillary-ISF circulation under both physiological and pathological situations. The same effect of high outflow, akin to venous, pressure is obtained by adding more fluid simulating volume expansion of the vascular system flooding the ISF space inducing combination of shock and ISF edema seen in MVOD/F.

Amazingly enough, on removing the narrow inlet, the $G$ tube behaves as Poiselle's tube of uniform diameter; the side pressure on the wall turns positive all along the tube, so does the pressure in the chamber that retains more volume, and the G-C circulation becomes sluggish. This mimics what is described as the "hyperdynamic circulation" seen in ARDS or MVOD/F! On inducing changes in the proximal and distal pressures, orifice diameter and volume expansion of the circulation, such pressure and volume changes may simulate and explain all types of known shock and that 
of the ARDS or MVOD/F. The G tube allows observer to visualize the phenomenon explaining the real capillary-ISF circulation and understand the involved forces under physiological conditions as well as the disturbances occurring under the pathological conditions of shock and MVOD/F. It is the only explanation of a true capillary-ISF circulation that can replace the faulty Starling' law

\section{How to Investigate VOS In ARDS?}

Quite simply measure the volume of infused fluid minus the loss of urine during the surgical procedure or shock resuscitation and/or monitor the change in the body weight of the patient with correlation to the clinical picture of ARDS. Investigating ARDS must start at the time of major surgery or fluid resuscitation.

\section{Conclusion}

Critical analysis of the reviewed literature revealing multiple errors and misconceptions on fluid therapy are reported and corrected. The reasons why Starling's law is wrong are discussed and a replacement based on the hydrodynamics of the $G$ tube is presented. The ARDS is induced by VOS that occur at the time of surgery or fluid resuscitation.

\section{References}

1. Ashbaugh DG, Bigelow DB, Petty TL, Levine BE (1967) Acute respiratory distress in adults. Lancet ii 2(7511): 319-323.

2. Wheeler A, Bernard G (2007) Acute lung injury and the acute respiratory distress syndrome. Lancet 369: 1553-1565.

3. Jacob M, Chappel D, Rehm M (2007) Clinical update: Perioperative fluid management. Lancet 369(9578): 1984-1986.

4. Starling EH (1886) Factors involved in the causation of dropsy. Lancet ii: 1266-1270, 1330-1334 and 1406-1414.

5. Ghanem AN, Ward JP (1990) Osmotic and metabolic sequelae of volumetric overload in relation to the TURP syndrome. Br J Uro 66(1): 71-78.

6. Ghanem AN (2001) Magnetic field-like fluid circulation of a porous orifice tube and relevance to the capillary-ISF fluid circulation: Preliminary report. Medical Hypotheses 56(3): 325-334.

7. Ghanem SA, Ghanem KA, Ghanem AN (2017) Volumetric Overload Shocks in the Patho-Etiology of the Transurethral Resection of the Prostate (TURP) syndrome and acute dilution hyponatraemia: the clinical evidence based on prospective clinical study of 100 consecutive TURP patients. Surg Med Open Access J 1(1).

8. Ghanem KA, Ghanem AN (2017) Volumetric overload shocks in the patho-etiology of the transurethral resection prostatectomy syndrome and acute dilution hyponatraemia: The clinical evidence based on 23 case series. Basic Research Journal of Medicine and Clinical Sciences 6(4): 35-43.

9. Pindoria N, Ghanem SA, Ghanem KA, Ghanem AN (2017) Volumetric overload shocks in the patho-etiology of the transurethral resection prostatectomy syndrome and acute dilution hyponatraemia. Integr Mol Med.

10. Ghanem KA, Ghanem AN (2017) The physiological proof that starling's law for the capillary-interstitial fluid transfer is wrong: Advancing the Porous Orifice (G) Tube phenomenon as replacement. Open Acc Res Anatomy 1(2).

11. Ghanem KA, Ghanem AN (2017) The proof and reasons that Starling's law for the capillary-interstitial fluid transfer is wrong, advancing the hydrodynamics of a porous orifice $(G)$ tube as the real mechanism. Blood, Heart and Circ 1(1): 1-7.

12. Ghanem AN, Ghanem SA (2016) Volumetric overload shocks: Why is starling's law for capillary interstitial fluid transfer wrong? the hydrodynamics of a porous orifice tube as alternative. Surgical Science 7: 245249.

13. Webster NR (1999) Monitoring the critically ill patient. J R Coll Surg Edin 44(6): 386-393.

14. Folkow B, Neil E (1971) Circulation. Oxford University Press: London, pp. 1-125.

15. Keele CA, Neil E, Joels N (1982) Sampson Wright Applied Physiology. $\left(13^{\text {th }}\right.$ edn), Oxford University Press; Oxford, USA.

16. Guyton AC (1986) Textbook of Medical Physiology. An HBJ International ( $7^{\text {th }}$ edn), WB Saunders Company. Philadelphia London. 19: 221.

17. (1998) Cochrane Injuries Group Albumen Reviewers. Human albumen administration in critically ill patients: systemic review of randomized controlled trials. Why albumen may not work. Br Med J 317(7153): 235240 .

18. Renkin EM (1986) Some consequences of capillary permeability to macromolecules: Starling's hypothesis reconsidered. Am J Physiol (Heart Circ Physiol) 250(5 Pt 2): H706-H710.

19. Pappenheimer JR, Soto RA (1948) Effective osmotic pressure of plasma proteins and other quantities associated with capillary circulation in the hind limbs of cats and dogs. Am J Physiol 152(3): 471-491.

20. Guyton AC, Coleman TG (1968) Regulation of interstitial fluid volume and pressure. Annals New York Academy of Sciences 150(3): 537-547.

21. Calnan JS, Pflug JJ, Chisholm GD, Taylor LM (1972) Lymphatic surgery. Proceedings Royal Soc Med 65: 715-719.

22. Harrison III RH, Boren JS, Robinson JR (1956) Dilutional hyponatremic shock: another concept of the transurethral prostatic reaction. J Urol 75(1): 95-110.

23. Danowski TS, Winkler AW, Elkington JR (1946) The treatment of shock due to salt depression; comparison of isotonic, of hypertonic saline and of isotonic glucose solutions. J Clin Invest 25(1): 130-138.

24. Rhodin JA (1967) The ultra-structure of mammalian arterioles and precapillary sphincters. J Ultrastructure Research 18(1-2): 181-223.

25. Karnovesky MJ (1967) The ultra-structural basis of capillary permeability studied with peroxidase as a tracer. J Cell Biol 35(1): 213-236.

26. Hendry EB (1962) The osmotic pressure and chemical composition of human body fluids. Clinical Chemistry 8(3): 246-265. 
(c) (i) Creative Commons Attribution 4.0 International License

For possible submissions Click Here

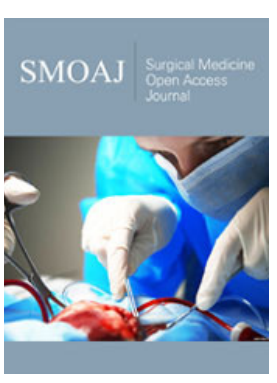

Surgical Medicine Open Access Journal

\section{Benefits of Publishing with us}

- High-level peer review and editorial services

- Freely accessible online immediately upon publication

- Authors retain the copyright to their work

- Licensing it under a Creative Commons license

- Visibility through different online platforms 